P04.14.343

Acta Cryst. (2008). A64, C338

\section{A novel octamer structure of bacterial cellulose synthesis component AxCesD}

Min $\mathrm{Yao}^{1}$, SongQing $\mathrm{Hu}^{1,3}$, Kenji Tajima ${ }^{2}$, YongQui Gao ${ }^{1}$, Naoki Sunagawa $^{2}$, Yong Zhou' ${ }^{1}$, Daisuke Shimura ${ }^{2}$, Masanobu Munekata ${ }^{2}$, Isao Tanaka ${ }^{1}$

${ }^{1}$ Hokkaido University, Faculty of Advanced Life Science, Kita-ku, Kita-10, Nishi-8, Sapporo, Hokkaido, 060-0810, Japan, ${ }^{2}$ Graduate School of Engineering, Hokkaido University 060-8628, Japan, ${ }^{3}$ Institute of Light Chemical Engineering, South China University of Technology, Guangzhou 510640, Guangdong, China, E-mail:yao@castor.sci.hokudai.ac.jp

Cellulose, a linear homopolymer of D-glucopyranose rings (DGPRs) with $\beta-1,4$ linkages is an affluent biopolymer in the nature, that is used in production of many commercial materials such as paper, films, textiles, explosive, and food additives. Although previous studies showed that cellulose can be produced by many organisms including plants, algae, some bacteria, even some animals, the aspects of cellulose biosynthesis are not yet fully understood. Cellulose produced by bacteria such as Gram-negative bacterium Acetobacter xylinum (A. xylinum) is called bacterial cellulose (BC), which has the different structural and physical characters from those of cellulose produced by plants. A. xylinum synthesizes cellulose from UDP-glucose and forms cellulose fibril, and has a BC synthase operon, AxCes that includes three (axces $A B$, axces $C$, and $\operatorname{axces} D)$ or four (axces $A$, axces $B$, axces $C$, and axces $D$ ) genes encoding a transmembrane protein complex called terminal complex (TC). The $\mathrm{AxCes} \mathrm{A}$ and $\mathrm{AxCesB}$ (or $\mathrm{AxCes} \mathrm{AB}$ ) are identified as the catalytic subunits, while the $\mathrm{AxCes} C$ is suggested to form the pore in the cell walls for export of cellulose fibril. The AxCesD protein is the smallest member in $\mathrm{BC}$ synthase components, and responsible for celluloses crystallization. Moreover, it has been reported that lack of the axces $D$ gene product resulted in a $40 \%$ reduction in the rates of bacterial cellulose synthesis. We have solved the structures of AxCesD in three forms, $\mathrm{N}$-terminus His-tagged and $\mathrm{C}$-terminus His-tagged proteins, and one complex with $\beta$-D-glucopyranose ring ( $\beta$-DGPR). AxCesD exits as an octamer forming a molecular ring. Combining with biochemical experiment, this molecular ring may play the role of gate for forming cellulose fibril.

Keywords: crystal structures of new compounds, cellulose biosynthesis, macromolecular assemblies

\section{P04.14.344}

Acta Cryst. (2008). A64, C338

\section{Structure of Narcissus pseudonarcissus lectin complex with mannobiose at $1.7 \AA$ resolution, FORM II}

\section{Suheyla Ozbey $^{1,3}$, Markus K Sauerborn ${ }^{2,3}$, Pierre J Rizkallah ${ }^{3}$}

${ }^{1}$ Hacettepe University, Engineering of Physics, Hacettepe University, Engineering of Physics, Faculty of Engineering, Ankara, Beytepe, 06800, Turkey, ${ }^{2}$ Kristallographie Gruppe Max-Delbrück-Centrum für Molekulare Medizin, Robert-Rössle-Strasse 10 D-13092 Berlin, Germany, ${ }^{3}$ Synchrotron Radiation Dept, CCLRC Daresbury Laboratory, Warrington, WA4 4AD, UK, E-mail:sozbey@hacettepe.edu.tr

A second preparation of crystals of the daffodil lectin in complex with alpha-1,3 mannobiose revealed a new stacking pattern, compared with the previously determined structure, 1NPL. Tetramers observed in the old, Form I, preparation were packed in layers that repeated by unit cell repetition normal to the layers (the $\mathrm{z}$ direction). Form II also has the same layering pattern, except that the repeat in the direction normal to the layers was shifted by $2.7 \AA$ in the plane. The result was a doubling of the $\mathrm{z}$ cell dimension, and the loss of the binding ability in the fourth binding site, at the interface between layers. Another crystallographic consequence was the incidental, purely translational, non-crystallographic symmetry, which gave faint bands in the diffraction pattern. The reason for the stacking loss of register is unclear, although the sample preparation was the same as before. Interaction energies across the interfaces were also calculated, in an attempt to clarify the differences.

Keywords: lectin crystallography, protein-saccharide interactions, molecular recognition

\section{P04.14.345}

Acta Cryst. (2008). A64, C338

\section{Crystal structure of a lectin from the octocoral}

Akiko Kita $^{1}$, Mitsuru Jimbo ${ }^{2}$, Yukio Morimoto ${ }^{1}$, Ryuichi Sakai ${ }^{2}$, Hisao Kamiya ${ }^{2}$, Kunio Miki ${ }^{3}$

${ }^{1}$ Research Reactor Institute, Kyoto University, Kumatori-Cho, SennanGun, Osaka, 590-0494, Japan, ${ }^{2}$ Department of Marine Biosciences, School of Fisheries Sciences, Kitasato University, Ofunato, Iwate 022-0101, Japan, ${ }^{3}$ Department of Chemistry, Graduate School of Science, Kyoto University, Kyoto 606-8502, Japan, E-mail:kita@rri.kyoto-u.ac.jp

Lectins are sugar-binding proteins or glycoproteins that recognize specific carbohydrate structures and agglutinate various types of animal cells. In marine animals, lectins are believed to contribute as non-self recognition factors to the defence mechanism. Interestingly, it has been theorized that some lectins from marine animals mediate the interaction between symbiont and host. SLL-2 is a D-galactose binding lectin isolated from an octocoral, Sinularia lochmodes. It was found that SLL-2 was distributed densely on the surface of symbiotic dinoflagellate Symbiodinium sp. cells. Recently, SLL-2 was indicated to transform free-swimming stage Symbiodinium cells into non-motile stage Symbiodinium cells and keep them in their nonmotile stage (K. Koike, et al., 2004). These results show that SLL-2 is a chemical cue in the symbiosis between dinoflagellates and coral. The three-dimensional structure of SLL-2 will provide information about the symbiosis mechanism. The purified SLL-2 was crystallized and the best crystals were obtained from the solutions containing 2-methyl-2,4-pentanediol as a precipitant and calcium formate as an additive reagent without the presence of any sugars. The structure of SLL-2 was solved by the molecular replacement method using atomic coordinates of HPA lectin (PDB code: $2 \mathrm{ccv}$ ) as a search model. Three SLL-2 monomers form a trimer around a non-crystallographic 3-fold axis, and two trimers formed a hexmeric assembly using hydrogen bonds of $\beta$-strand $(\beta 1 \mathrm{~N})$ from each monomer. The structure of SLL-2 monomer shows the $\beta$-sandwich lectin fold with six $\beta$-strands ( $\beta 1-\beta$ 6 ). The sites of $\mathrm{N}$-glycosylation and sugar binding were identified in the crystal structure.

Keywords: protein crystallization, protein structure analysis, lectins

\section{P04.14.346}

Acta Cryst. (2008). A64, C338-339

X-ray crystallographic analysis of galectin LEC-8 from Caenorhabditis elegans

Takashi Itagaki $^{1,5}$, Chiaki Endo ${ }^{1,5}$, Shun-ichi Kidokoro ${ }^{5}$, Yoichiro Arata $^{2}$, Jun Hirabayashi ${ }^{3}$, Ken-ichi Kasai ${ }^{4}$,

Takamasa Nonaka

${ }^{1}$ Iwate Medical University, School of Pharmacy, 2-1-1 Nishitokuta, 